\title{
Prioritized channel allocation-based dynamic spectrum access in cognitive radio sensor networks without spectrum handoff
}

\author{
Jong-Hong Park and Jong-Moon Chung* (D)
}

\begin{abstract}
In this article, a dynamic spectrum access (DSA) method is proposed to perform dynamic prioritized channel allocation in cognitive radio sensor networks (CRSNs) that do not support spectrum handoff. The proposed CRSN supports primary users (PUs) and two types of secondary users (SUs), where one type of SU data traffic has a higher priority. The proposed prioritized channel allocation (PCA) scheme was designed to provide adaptive spectrum access control for CRSNs supporting SU prioritized data types. Compared to the existing DSA schemes, SNO and DSA-C2, the proposed PCA scheme shows an advantage in throughput and fairness in supporting SU prioritized traffic.
\end{abstract}

Keywords: Cognitive radio, Dynamic spectrum access, Prioritized channel allocation, Multi-dimensional Markov chain

\section{Introduction}

Cognitive radio (CR) sensor networks (CRSNs) have been proposed to improve the channel utilization of wireless networks suffering from serious shortage of radio spectrum. In CRSNs, licensed users use assigned channels as primary users (PUs). On the other hand, each sensor node which does not have a license to access a channel is a secondary user (SU), where SUs attempt to access a channel only if it is not used by a PU. In recent studies, dynamic spectrum access (DSA) strategies for CRSNs have been considered [1-6]. DSA channel assignment schemes can be categorized as centralized, distributed, and decentralized [2]. Centralized schemes have a central node that collects channel and link information from the sensor nodes [7-9]. The collected information is used to execute the channel assignment algorithm to determine the channel assignments for all of the links in the wireless sensor network. In distributed schemes, each node calculates the channel environment and condition of its local links and selects the appropriate channel considering local information [10-12]. A decentralized DSA can be operated in cluster-based wireless sensor networks, where a cluster head performs the intra-cluster channel

*Correspondence: jmc@yonsei.ac.kr

School of Electrical and Electronic Engineering, Yonsei University, 03722 Seoul, Republic of Korea assignment in a centralized form and computes the inter-cluster channel assignment in a distributed manner $[13,14]$. The distributed and decentralized DSA schemes have an advantage in not requiring an additional central control system. However, since the nodes in the CRSN only use local information, distributed and decentralized DSA schemes may not result in optimal results [2]. In addition, a majority of existing research on DSA for CRSNs assume that SUs are equipped with spectrum handoff $(\mathrm{SHO})$ functionality. As $\mathrm{SHO}$ requires additional operations (e.g., scanning for open channels, transmitterreceiver handshake, etc.), CR devices supporting $\mathrm{SHO}$ will consume additional energy and also a CR channel controller is needed [15-17]. Due to these reasons, there are CRSNs that do not have SHO functionality. In this article, a prioritized channel allocation (PCA) scheme is proposed to enhance the performance of CRSNs that do not support SHO functionality in a centralized DSA scheme. The proposed CRSN scheme supports PUs and two types of CR SUs, where one type of SU data traffic has a higher priority over the other type. Priority distinction in SU data packets was found needed in many CRSN applications where control/management messages/data and alarm information requires higher priority support from the CRSN. Therefore, the proposed PCA scheme was designed to provide adaptive spectrum access control for CRSNs supporting prioritized SU data types. 
In the proposed PCA scheme, the entire sub-channel was designed to be divided for each class of SUs without SHO functionality. After the allocation of PUs, each SU is allocated. By separating sections of sub-channels for SUs and assigning sub-channels from the highest number, the transmission success rate and connection sustaining rate of SUs with higher priority can be optimized.

The state transitions of the proposed PCA scheme are modeled as a multi-dimensional Markov chain with three state variables. Based on the state transition modeling, the blocking probability (BP), forced termination probability (FTP), and the call completion rate (CCR) are analyzed for the SUs with and without priority service support in the CRSN.

The rest of this paper is organized as follows. In Section 2, the system model and the proposed PCA scheme for centralized CRSNs are presented. In Section 3, the analytical models based on Markov chains with respect to the proposed PCA scheme are provided. In Section 4, the performance measures including the BP, FTP, and CCR for both $\mathrm{SU}_{1}$ and $\mathrm{SU}_{2}$ are derived. In Section 5, the numerical and simulation results under the proposed PCA scheme are presented. Finally, Section 6 gives the concluding remarks.

\section{System model}

Figure 1 illustrates the $C R$ system that consists of $M$ licensed channels. Each licensed band is further divided into $N$ sub-channels for CR SUs, therefore, there are a maximum of $M \times N$ sub-channels that can be used by the SUs. The CRSN supports PUs and two types of CR SUs, where $\mathrm{SU}_{1}$ represents the SUs with higher priority, and $\mathrm{SU}_{2}$ represents the $\mathrm{SUs}$ with lower priority. In addition, the arrival of $\mathrm{PU}, \mathrm{SU}_{1}$, and $\mathrm{SU}_{2}$ packets are assumed to be Poisson processes with arrival rates $\lambda_{p}, \lambda_{s 1}$, and $\lambda_{s 2}$, respectively, and the corresponding expected service times are exponentially distributed with rates $\mu_{p}, \mu_{s 1}$, and $\mu_{s 2}$, respectively [7-9][18-20], which is the same model used in the analysis of the DSA scheme with no buffer for the centralized CRSN (DSA-C2) [7] and sequential number ordering (SNO) scheme [9] as well as other schemes dealing with prioritized traffic [8].

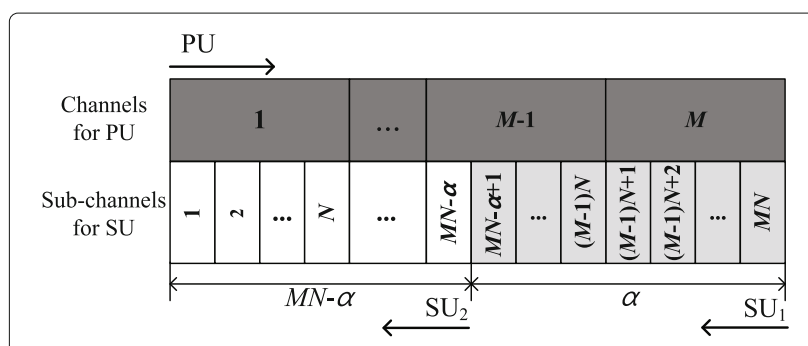

Fig. 1 System model
In the proposed PCA scheme, the sub-channels are divided into two sections. One section is for $\mathrm{SU}_{1}$, where $\alpha(0<\alpha<M N)$ sub-channels are assigned among $M N$ sub-channels. The other section is for $\mathrm{SU}_{2}$ users, where up to $M N-\alpha$ sub-channels can be assigned. In the proposed scheme, channel allocation of PUs are assigned in an ascending sequential order (i.e., $1,2, \ldots, M$ ) and the $\mathrm{SU}_{1} \mathrm{~s}$ scan and use the $\mathrm{CR}$ sub-channels in a descending sequential order from $M N$ to $M N-\alpha+1$ (i.e., $M N, M N-$ $1, \ldots, M N-\alpha+1)$. In addition, the $\mathrm{SU}_{2} \mathrm{~s}$ scan and use the $\mathrm{CR}$ sub-channels in a descending sequential order from $M N-\alpha$ to 1 (i.e., $M N-\alpha, M N-\alpha-1, \ldots, 1$ ).

The CRSN that applies the proposed PCA scheme is fully connected, which means that all SUs observe the same channel status. It is assumed for simplicity that a PU call requires one channel, whereas a SU call requires one sub-channel. In addition, a common control channel is assumed to exist for coordination among the $\mathrm{SU}_{1} \mathrm{~s}$ and $\mathrm{SU}_{2} \mathrm{~S}$, and perfect sensing is assumed to detect the PUs and SUs activity. The bandwidth requirement of $\mathrm{SU}_{1}$ and $\mathrm{SU}_{2}$ is assumed to be identical, and there is no specific geo-location requirement of $\mathrm{SU}_{1} \mathrm{~s}$ and $\mathrm{SU}_{2} \mathrm{~S}$.

The DSA scheme also needs to resolve the conflicts between competitive SUs to establish fairness in spectrum sharing. Thus, in the design of the DSA scheme for CRSNs, consideration of fairness among multiple resource-competitive sensors and priorities of sensor data is maintained. In throughput maximization schemes, some sensor nodes may need to be assigned additional channel resources than others, resulting in partial starvation [15]. Therefore, for optimal spectrum access control in both throughput and fairness of prioritized traffic, the proposed PCA scheme aims at allocating the channel region to be used by higher priority $\mathrm{SU}_{1}$ calls with a following constraint on the fairness indicator (FI) [21]

$$
\mathrm{FI}=\frac{\left(T_{1}+T_{2}\right)^{2}}{2\left(T_{1}^{2}+T_{2}^{2}\right)}
$$

where $T_{1}$ and $T_{2}$ are the CCR of $\mathrm{SU}_{1}$ and $\mathrm{SU}_{2}$ calls, respectively. The CCR of $\mathrm{SU}_{1}$ and $\mathrm{SU}_{2}$ are the mean number of calls which is determined by the BP and FTP of $\mathrm{SU}_{1}$ or $\mathrm{SU}_{2}$, described in detail in Section 4. FI is a measure of how fair the channel resources are shared among $\mathrm{SU}_{1} \mathrm{~s}$ and $\mathrm{SU}_{2} \mathrm{~S}$ [21]. The lower bound of $\mathrm{FI}, \varepsilon$, is a minimum required value of fairness and FI is upper bounded by 1 . A higher FI value is an indicator of a fairer network.

Let $\rho_{p}=\lambda_{p} / \mu_{p}, \rho_{s 1}=\lambda_{s 1} / \mu_{s 1}$, and $\rho_{s 2}=\lambda_{s 2} / \mu_{s 2}$ be the channel utilization of $\mathrm{PU}, \mathrm{SU}_{1}$, and $\mathrm{SU}_{2}$, respectively. The average number of $P U_{s}, \mathrm{SU}_{1} \mathrm{~s}$, and $\mathrm{SU}_{2} \mathrm{~s}$ in the system in steady-state can be estimated as $U_{p}=\rho_{p} /\left(1-\rho_{p}\right), U_{s 1}=$ $\rho_{s 1} /\left(1-\rho_{s 1}\right)$, and $U_{s 2}=\rho_{s 2} /\left(1-\rho_{s 2}\right)$, respectively $[22,23]$. Based on the estimations, it is predicted that $\left(M-\left[U_{p}\right]\right) N$ channels can be used by the SUs (where [.] denotes an integer round function) where the number of allocated 
channels for $\mathrm{SU}_{1}$ (i.e., $\alpha$ ) can be derived based on the estimated channel values using

$$
\alpha=\left[\left(M-\left[U_{p}\right]\right) N \frac{U_{s 1}}{U_{s 1}+U_{s 2}}\right] .
$$

\section{Markov chain model}

The proposed PCA scheme is designed based on a multidimensional Markov chain model with three-state variables in set form $(i, j, k)$, where, $i, j$, and $k$ are integers that present the number of PUs, $\mathrm{SU}_{1} \mathrm{~s}$, and $\mathrm{SU}_{2} \mathrm{~s}$ occupying the channels, respectively. It is required that $i N+j+k \leq M N$, thus the state space $\mathrm{S}$ is given by

$$
S=\{(i, j, k) \mid 0 \leq i \leq M ; 0 \leq j \leq \alpha ; 0 \leq k \leq M N-\alpha\}
$$

where the state transition analysis will use probability notations based on $p$ as the quotient when $\alpha$ is divided by $N, q$ is the remainder (i.e., $\alpha=p N+q, 0 \leq p<M, 0 \leq q<$ $N)$. In addition, $p^{\prime}$ is the quotient when $j$ is divided by $N, q^{\prime}$ is the remainder (i.e., $j=p^{\prime} N+q^{\prime}, 0 \leq p^{\prime}<M, 0 \leq q^{\prime}<N$ ). A multi-dimensional Markov chain is used to analyze the performance of the proposed scheme, where $P(i, j, k)$ is the state probability of the state $(i, j, k)$, and $\phi(i, j, k)$ is defined as an indication function that equals 1 only if state $(i, j, k)$ is feasible, and is 0 else wise. Fig. 2 illustrates the Markov chain model of the proposed PCA scheme.

3.1 Case 1-1: from state $(i, j, k)$ to other states when $k=0$ 1) PU requests service When a PU requests for service, one of the three following transitions occurs:

- If $i N+j=M N$, the ongoing $N$ packets of $\mathrm{SU}_{1}$ will be forced to terminate by the arrival of a PU. Then, a state transition $(i, j, 0) \rightarrow(i+1, j-N, 0)$ will occur with rate $\phi(i+1, j-N, 0) \lambda_{p}$.

- If $(M-1) N<i N+j<M N$, and $j=p^{\prime} N+q^{\prime}$ $\left(0 \leq p^{\prime} \leq M, 0 \leq q^{\prime}<N\right)$, the arrival of a PU will drop the ongoing $q^{\prime}$ packet(s) of $\mathrm{SU}_{1}$. Accordingly, state transition $(i, j, 0) \rightarrow\left(i+1, p^{\prime} N, 0\right)$ will occur with rate $\phi\left(i+1, p^{\prime} N, 0\right) \lambda_{p}$.

- If $i N+j \leq(M-1) N$, the arrival of a PU will be assigned one channel without any $\mathrm{SU}_{1}$ user being forced to terminate. In this case, state transition $(i, j, 0) \rightarrow(i+1, j, 0)$ will occur with rate $\phi(i+1, j, 0) \lambda_{p}$.

2) PU completes service In this case, state transition $(i, j, 0) \rightarrow(i-1, j, 0)$ will occur with rate $\phi(i-$ $1, j, 0) i \mu_{p}$. 3) $S U_{1}$ Requests Service In case of $k=0$, $\mathrm{SU}_{1}$ can request for service and access an unoccupied sub-channel if $i N+j<M N$. In this case, state transition $(i, j, 0) \rightarrow(i, j+1,0)$ will occur with rate $\phi(i, j+1,0) \lambda_{s 1}$.

4) $S U_{1}$ completes service In this case, state transition $(i, j, 0) \rightarrow(i, j-1,0)$ will occur with rate $\phi(i, j-1,0) j \mu_{s 1}$.
5) $\mathrm{SU}_{2}$ requests service $\mathrm{SU}_{2}$ can request for service and access an unoccupied sub-channel when both $i N+j<M N$ and $i<M-p$. In this case, state transition $(i, j, 0) \rightarrow(i, j, 1)$ will occur with rate $\phi(i, j, 1) \lambda_{s 2}$.

3.2 Case 1-2: from other states to state $(i, j, k)$ when $k=0$ 1) PU requests service When a PU requests for service, one of the three following transitions can occur:

- If $i N+j=M N$ and $p^{\prime}=p$, the following states can transfer to state $(i, j, 0)$ with rate $\phi\left(i-1, j^{\prime}, k^{\prime}\right) \lambda_{p}$ based on $\left(i-1, j^{\prime}, k^{\prime}\right) \rightarrow(i, j, 0)$ where $j^{\prime}+k^{\prime} \leq N, 0 \leq j^{\prime} \leq q$, and $0 \leq k^{\prime} \leq N-q$. In this case, $j^{\prime} \mathrm{SU}_{1} \mathrm{~s}$ and $k^{\prime} \mathrm{SU}_{2} \mathrm{~S}$ suffer from forced termination when a PU accesses the channel.

- If $i N+j=M N$ and $p^{\prime}<p$, the following states can transfer to state $(i, j, 0)$ with rate $\phi(i-1, j+n, 0) \lambda_{p}$ based on $(i-1, j+n, 0) \rightarrow(i, j, 0)$ where $0 \leq n \leq N$. In this case, only $n \mathrm{SU}_{1}(\mathrm{~s})$ suffer(s) from forced termination when a PU accesses the channel.

- If $i N+j<M N$, the arriving PU will be assigned one channel without any $\mathrm{SU}_{1}$ and $\mathrm{SU}_{2}$ being forced to terminate. In this case, state transition with rate $\phi(i-1, j, 0) \lambda_{p}$ will result in $(i-1, j, 0) \rightarrow(i, j, 0)$.

2) PU completes service In this event, there is only one possibility for which the system will transit to state $(i, j, k)$ with rate $\phi(i+1, j, 0)(i+1) \mu_{p}$, when $i N+j \leq(M-1) N$, which is $(i+1, j, 0) \rightarrow(i, j, 0)$.

3) $S U_{1}$ requests service In case of $k=0$, this event results in a transition $(i, j-1,0) \rightarrow(i, j, 0)$ with rate $\phi(i, j-1,0) \lambda_{s 1}$.

4) $S U_{1}$ completes service When $i N+j<M N$, state transition $(i, j+1,0) \rightarrow(i, j, 0)$ will occur with rate $\phi(i, j+1, k)(j+$ 1) $\mu_{s 1}$.

5) $S U_{2}$ completes service $\mathrm{SU}_{2}$ can request service and access an unoccupied sub-channel for the case when both $i N+j<M N$ and $i<M-p$ are satisfied. In this case, the state transition $(i, j, 1) \rightarrow(i, j, 0)$ will occur with rate $\phi(i, j, 1) \mu_{s 2}$.

3.3 Case 2-1: from state $(i, j, k)$ to other states when $k>0$ 1) $P U$ requests service When a PU requests a service, one of the five following transitions occurs:

- If $i N+\alpha+k=M N$ and $i<M-p-1$, the arrival of a PU will results in a drop of the ongoing $N$ packets of $\mathrm{SU}_{2}$ without any $\mathrm{SU}_{1}$ being forced to terminate.

Then, there is only one state to which the system will transfer to (based on rate $\phi(i+1, j, k-N) \lambda_{p}$ ), which is $(i, j, k) \rightarrow(i+1, j, k-N)$.

- If $i N+\alpha+k=M N$ and $i=M-p-1$, the arrival of a PU will results in a drop of all ongoing packets of $\mathrm{SU}_{2}$. In addition, if $j>p n$, then $(j-p n)$ packets of $\mathrm{SU}_{1}$ will be forced to terminate. Otherwise, there is no packet of $\mathrm{SU}_{1}$ to be dropped. Therefore, the state 


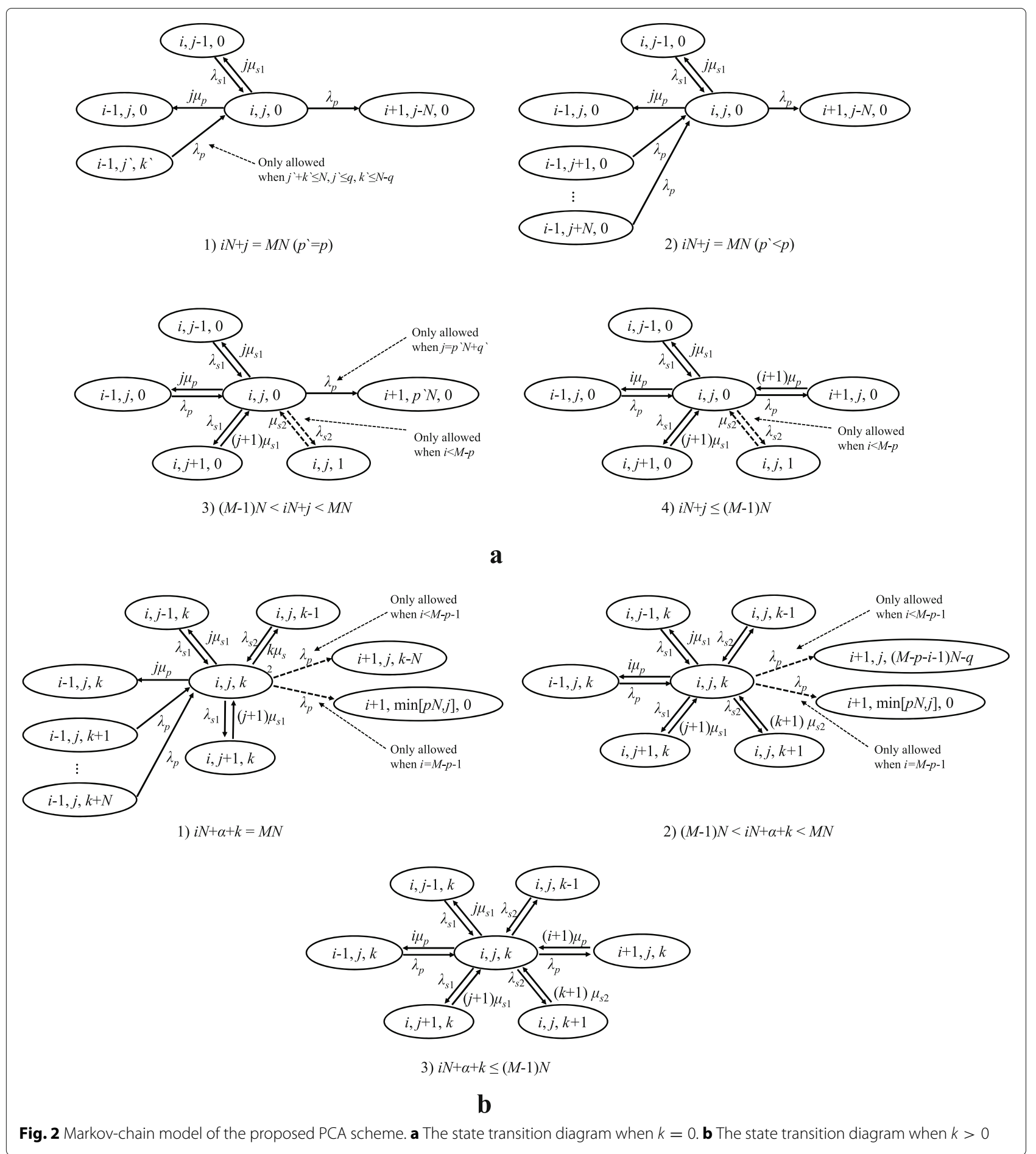

transition $(i, j, k) \rightarrow(i+1, \min [p N, j], 0)$ will occur with rate $\phi(i+1, \min [p N, j], 0) \lambda_{p}$.

- If $(M-1) N<i N+j<M N$ and $i<M-p-1$, the arrival of a PU will drop the ongoing packet(s) of $\mathrm{SU}_{2}$ without any $\mathrm{SU}_{1}$ being forced to terminate. Then, the state transition $(i, j, k) \rightarrow(i+1, j,(M-p-i-1) N-q)$ will occur with rate $\phi(i+1, j,(M-p-i-1) N-q) \lambda_{p}$.
- If $(M-1) N<i N+j<M N$ and $i=M-p-1$, the arrival of a PU will drop all ongoing packets of $\mathrm{SU}_{2}$. In addition, if $j>p n$, then $(j-p n)$ packets of $\mathrm{SU}_{1}$ will be forced to terminate. Otherwise (i.e., $j \leq p n$ ), no packet of $\mathrm{SU}_{1}$ needs to be dropped. Therefore, the state transition $(i, j, k) \rightarrow(i+1, \min [p N, j], 0)$ will occur with rate $\phi(i+1, \min [p N, j], 0) \lambda_{p}$. 
- If $i N+j \leq(M-1) N$, the arrival of a PU will be assigned one channel without any $\mathrm{SU}_{1}$ and $\mathrm{SU}_{2}$ being forced to terminate. In this case, state transition $(i, j, k) \rightarrow(i+1, j, k)$ will occur with rate $\phi(i+1, j, 0) \lambda_{p}$.

2) $P U$ completes service In this case, state transition $(i, j, k) \rightarrow(i-1, j, k)$ will occur with rate $\phi(i-1, j, k) i \mu_{p}$.

3) $S U_{1}$ requests service $S U_{1}$ can request for service and access an unoccupied sub-channel when both $i N+j+k<$ $M N$ and $j<\alpha$ are satisfied. In this case, state transition $(i, j, k) \rightarrow(i, j+1, k)$ will occur with rate $\phi(i, j+1, k) \lambda_{s 1}$.

4) $S U_{1}$ completes service In this case, state transition $(i, j, k) \rightarrow(i, j-1, k)$ will occur with rate $\phi(i, j-1, k) j \mu_{s 1}$.

5) $S U_{2}$ requests service $\mathrm{SU}_{2}$ can request for service and access an unoccupied sub-channel when $i N+\alpha+k<M N$. In this case, state transition $(i, j, k) \rightarrow(i, j, k+1)$ will occur with rate $\phi(i, j, k+1) \lambda_{s 2}$.

6) $S U_{2}$ completes service In this case, state transition $(i, j, k) \rightarrow(i, j, k-1)$ will occur with rate $\phi(i, j, k-1) k \mu_{s 2}$.

\subsection{Case 2-2: from other states to state $(i, j, k)$ when $k>0$} 1) PU requests service When a PU requests for service, one of the two following transitions can occur:

- If $i N+\alpha+k=M N$, with rate $\phi(i-1, j, k+n) \lambda_{p}$, the states based on $0 \leq n \leq N$ can transfer to state $(i, j, 0)$ in the form of $(i-1, j, k+n) \rightarrow(i, j, k)$. In this case, only $n \mathrm{SU}_{2}(\mathrm{~s})$ suffer(s) from forced termination when a PU accesses the channel.

- If $i N+\alpha+k<M N$, the arrival of a PU will be assigned one channel without any $\mathrm{SU}_{1}$ and $\mathrm{SU}_{2}$ being forced to terminate. In this case, state transition $(i-1, j, k) \rightarrow(i, j, k)$ will occur with rate $\phi(i-1, j, k) \lambda_{p}$.

2) $P U$ completes service In this event, there is only one possibility for which the system will transit to state $(i, j, k)$ with rate $\phi(i+1, j, 0)(i+1) \mu_{p}$, when $i N+\alpha+k \leq(M-1) N$, which is $(i+1, j, k) \rightarrow(i, j, k)$.

3) $S U_{1}$ requests service This event results in a transition with rate $\phi(i, j-1, k) \lambda_{s 1}$ from $(i, j-1, k) \rightarrow(i, j, k)$,

4) $S U_{1}$ completes service In this case, the state transition $(i, j+1, k) \rightarrow(i, j, k)$ will occur with rate $\phi(i, j+1, k)(j+1) \mu_{s 1}$.

5) $S U_{2}$ requests service In this case, state transition $(i, j, k-1) \rightarrow(i, j, k)$ will occur with rate $\phi(i, j, k-1) \lambda_{s 2}$.

6) $S U_{2}$ completes service $\mathrm{SU}_{2}$ can request service and access an unoccupied sub-channel based on the condition of $i N+j<M N$. In this case, state transition $(i, j, k+1) \rightarrow$ $(i, j, k)$ will occur with rate $\phi(i, j, k+1)(k+1) \mu_{s 2}$.

Figure 3 illustrates the proposed PCA mechanism. The channel is estimated based on the arrival rates and the service rates of the PUs, $\mathrm{SU}_{1} \mathrm{~s}$, and $\mathrm{SU}_{2} \mathrm{~S}$ including the number of channels and sub-channels, $M$ and $N$ to find the value of $\alpha$ in (2). The steady-state probability of each state

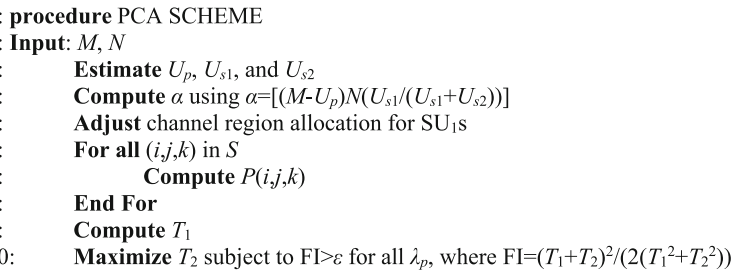

Fig. 3 The proposed PCA scheme

$P(i, j, k)$ is obtained from the multi-dimensional Markov chain [8]. The linear equation system needs to be constructed including the balance equations of all states in $S$ and the normalized equation. The normalized equation of the system is the equation that requires the sum of all feasible states to add up to 1 . From the linear equation system, the steady-state probability of each state $P(i, j, k)$ is computed and $T_{1}$ (i.e., CCR of $\mathrm{SU}_{1}$ ) is obtained. Finally, the CCR of $\mathrm{SU}_{2}$ (i.e., $T_{2}$ ) is maximized under the constraint. When channel variation is detected (i.e., $U_{p}, U_{s 1}$, or $U_{s 2}$ changes), the above process is repeated. In other words, if the arrival rate or service rate of any user changes, the proposed algorithm should be performed again to find the optimal value of $\alpha$ according to the channel condition.

\section{Performance analysis}

\subsection{Blocking probability (BP)}

$\mathrm{BP}$ is the probability that the arrival of a $\mathrm{SU}_{1}$ call or $\mathrm{SU}_{2}$ call is not allowed access to a channel due to all subchannels of $\mathrm{SU}_{1}$ or $\mathrm{SU}_{2}$ being fully occupied. $\mathrm{BP}$ of $\mathrm{SU}_{1}$ calls, denoted as $P_{B 1}$, can be expressed as

$$
P_{B 1}=\sum_{i=0}^{M-p-1} \sum_{k=0}^{M N-\alpha} P(i, \alpha, k)+\sum_{i=M-p}^{M} P(i,(M-i) N, 0)
$$

BP for $\mathrm{SU}_{2}$ calls, denoted as $P_{B 2}$, can be expressed as

$$
P_{B 2}=\sum_{i=0}^{M-p-1} \sum_{j=0}^{\alpha} P(i, j,(M-i) N-\alpha)+\sum_{i=M-p}^{M} \sum_{j=0}^{\alpha} P(i, j, 0)
$$

\subsection{Forced termination probability (FTP)}

FTP is the probability that an ongoing $\mathrm{SU}_{1}$ call or $\mathrm{SU}_{2}$ call is terminated by an incoming PU call. FTP for $\mathrm{SU}_{1}$ calls, denoted as $P_{F 1}$, can be expressed as

$$
\begin{aligned}
P_{F 1}= & \frac{\lambda_{p}}{\lambda_{s 1}\left(1-P_{B 1}\right)} \sum_{i=M-p}^{M-1} \sum_{q^{\prime}=1}^{N} q^{\prime} P\left(i,(M-1-i) N+q^{\prime}, 0\right) \\
& +\frac{\lambda_{p}}{\lambda_{s 1}\left(1-P_{B 1}\right)} \sum_{q^{\prime}=1}^{q} \sum_{k=0}^{M N-\alpha} q^{\prime} P\left(i, p N+q^{\prime}, k\right)
\end{aligned}
$$

Similarly, FTP for $\mathrm{SU}_{2}$ calls, $P_{F 2}$, can be expressed as 

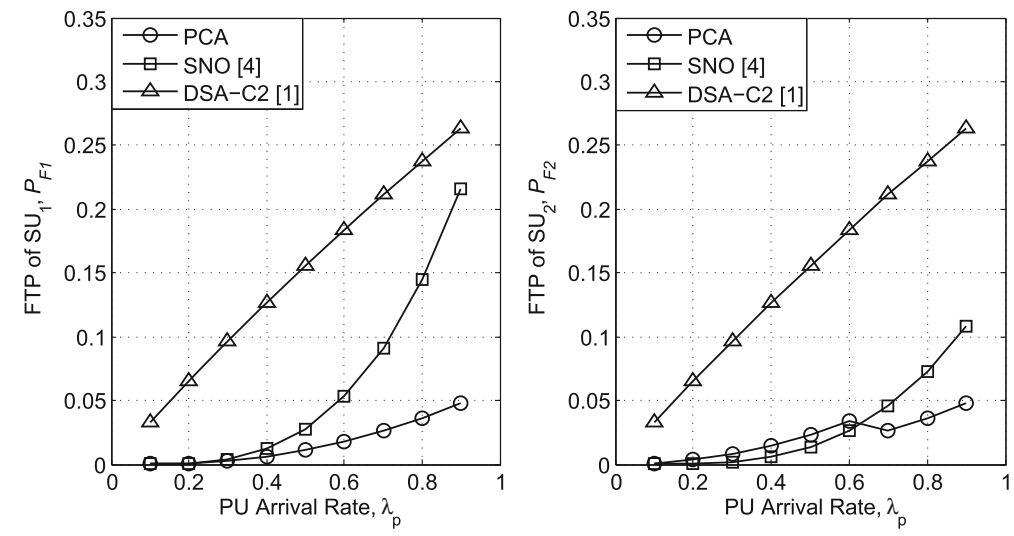

Fig. 4 Analysis of FTP of $\mathrm{SU}_{1}$ and $\mathrm{SU}_{2}$ versus the arrival rate of the PU

$$
\begin{aligned}
P_{F 2}= & \frac{\lambda_{p}}{\lambda_{s 2}\left(1-P_{B 2}\right)} . \\
& \sum_{i=0}^{M-p-2} \sum_{j=0}^{\alpha} \sum_{k^{\prime}=1}^{N} k^{\prime} P\left(i, j,(M-p-i-1) N-q+k^{\prime}\right) \\
& +\frac{\lambda_{p}}{\lambda_{s 2}\left(1-P_{B 2}\right)} \sum_{j=0}^{\alpha} \sum_{k=1}^{N-q} k P(M-p-1, j, k) .
\end{aligned}
$$

\subsection{Call completion rate (CCR)}

CCR is the mean number of calls that successfully complete their service, where $T_{1}$ and $T_{2}$ of (8) and (9) denote the $\mathrm{CCR}$ for $\mathrm{SU}_{1}$ and $\mathrm{SU}_{2}$ calls, respectively [7].

$$
\begin{aligned}
& T_{1}=\lambda_{s 1}\left(1-P_{B 1}\right)\left(1-P_{F 1}\right) \\
& T_{2}=\lambda_{s 2}\left(1-P_{B 2}\right)\left(1-P_{F 2}\right)
\end{aligned}
$$

\section{Numerical results}

The proposed PCA scheme is compared to two other DSA schemes supporting prioritized sensor data traffic in CRSNs, which are the sequential number ordering
(SNO) scheme [9], and the DSA scheme with no buffer for centralized CRSN (DSA-C2) [7]. Since the computational complexity is determined by the number of licensed channels $(M)$ and the number of sub-channels $(N)$, the proposed PCA scheme and the compared schemes (i.e., SNO and DSA-C2) in this paper have the same computational complexity [24]. The computational complexity of the SNO [9] and DSA-C2 [7] schemes are identical to $O\left(M^{2} N^{2}\right)$ and that of the proposed PCA scheme is $O\left((M N-\alpha)^{2}+\alpha^{2}\right)$, which results in the same complexity of $O\left(M^{2} N^{2}\right)$ as SNO and DSA-C2. Simulation is conducted for the case where all schemes do not use SHO while supporting two class prioritized traffic based on the settings of $M=3, N=6, \mu_{p}=2, \lambda_{s 1}=0.2, \lambda_{s 2}=0.4, \mu_{s 1}=\mu_{s 2}=1$, and $\varepsilon=0.9$.

Figure 4 shows the FTP of $\mathrm{SU}_{1}$ and $\mathrm{SU}_{2}$, and Fig. 5 shows the CCR of $\mathrm{SU}_{1}$ and $\mathrm{SU}_{2}$, respectively, for various values of PU arrival rates $\lambda_{p}$. In addition, Fig. 6 shows that the proposed PCA scheme satisfies the constraint $\mathrm{FI} \geq 0.9$, as $\alpha$ is adapted corresponding to the channel conditions. Increasing the arrival rate of the PU leads to an increase
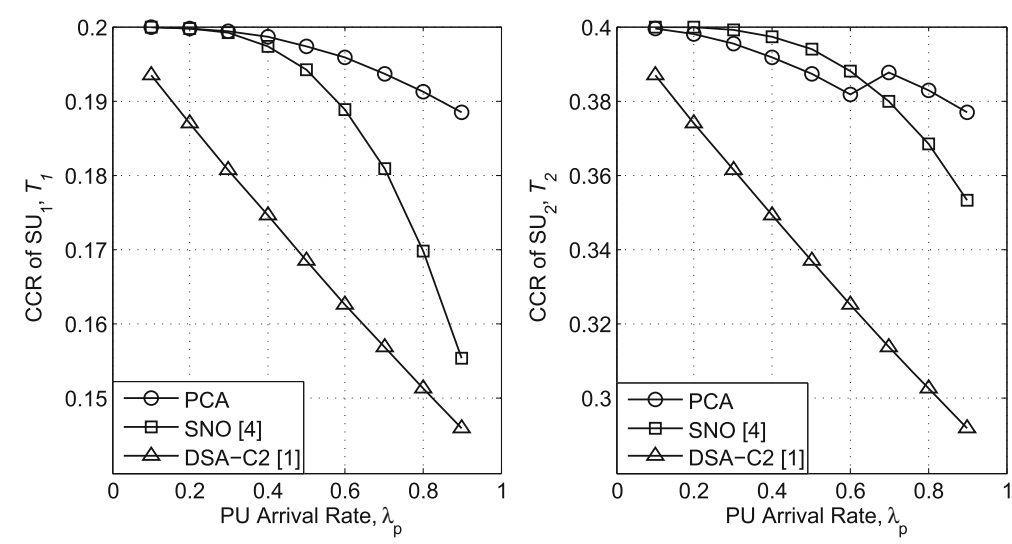

Fig. 5 Analysis of $C C R$ of $\mathrm{SU}_{1}$ and $\mathrm{SU}_{2}$ versus the arrival rate of the $\mathrm{PU}$ 


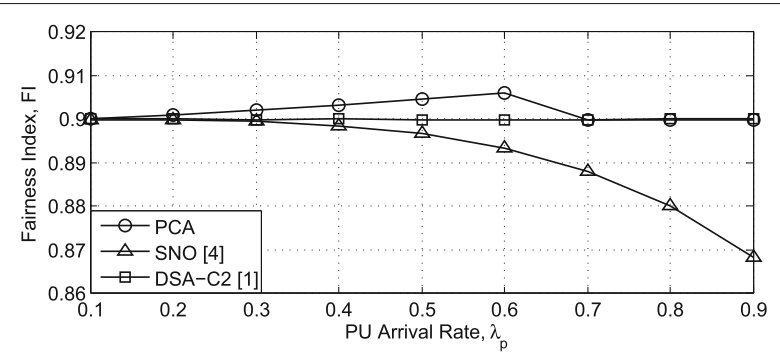

Fig. $6 \mathrm{FI}$ versus the arrival rate of the PU

in the FTP which results in a decrease in the performance of the CCR of both $\mathrm{SU}_{1}$ and $\mathrm{SU}_{2}$. However, when compared to the SNO and DSA-C2 schemes, respectively, the proposed PCA scheme results in $0 \sim 27.8$ and $4.28 \sim 35.81 \%$ performance gain in $\mathrm{CCR}$ of $\mathrm{SU}_{1} \mathrm{~S}$ when $\lambda_{p}$ increases from 0.1 to 0.9 . When $\lambda_{p}$ is smaller than 0.7 , the CCR of $\mathrm{SU}_{1}$ is satisfied when $\alpha=5$. When $\lambda_{p} \geq 0.7$, the CCR of $\mathrm{SU}_{1}$ is satisfied at $\alpha=3$ and $\mathrm{FI} \geq 0.9$. The $\mathrm{CCR}$ of $\mathrm{SU}_{2} \mathrm{~s}$ improves in performance gain compared to the SNO and DSA-C2 schemes in the range of $\lambda_{p}$ from 0.7 to 0.9 , due to the variation in $\alpha$ from 5 to 3 . The proposed PCA scheme aims to maximize the CCR of SUs with higher priority (i.e., $\mathrm{SU}_{1}$ ) preferentially, and maximize the CCR of SUs with lower priority (i.e., $\mathrm{SU}_{2}$ ) within the range that satisfies the FI constraint (i.e., $\mathrm{FI} \geq \varepsilon$ ). That is why the $\mathrm{CCR}$ of $\mathrm{SU}_{1}$ is maximized in all values of $\mathrm{PU}$ arrival rates while the CCR of $\mathrm{SU}_{2}$ is worse than that of the $\mathrm{SNO}$ scheme under low PU arrival rates as shown in Fig. 5. However, as shown in Fig. 6, the FI of the proposed PCA scheme satisfies the FI constraint, while that of the SNO scheme does not. The results show that the PCA scheme results in a gain in the $\mathrm{CCR}$ of $\mathrm{SU}_{1}$ and $\mathrm{SU}_{2}$ users when compared to the $\mathrm{SNO}$ and DSA-C2 schemes. This result comes from the fact that the proposed PCA scheme maximizes the $C C R$ of $\mathrm{SU}_{1}$ and $\mathrm{SU}_{2}$ while satisfying the fairness constraint.

\section{Conclusions}

In this article, an optimal CR channel allocation scheme that supports two types of prioritized sensor data types in CRSNs without SHO is proposed. The proposed PCA scheme was designed to provide optimal spectrum access control for CRSNs supporting heterogeneous SU data types. The performance analysis results show that when compared to the SNO scheme [9] and the DSA-C2 [7], the proposed PCA scheme can provide a significant advantage in FTP and CCR while satisfying the FI constraint in supporting SU prioritized traffic. For future work, improved modeling of DSA for prioritized traffic considering dynamic spectrum fragmentation/defragmentation and improved spatial channel reuse based on the geolocations of SUs will be conducted.

\section{Acknowledgments}

This work was supported by the ICT R\&D program of MSIP/ITP.

[R0126-16-1009, Development of Smart Mediator for Mashup Service and Information Sharing among ICBMS Platform].

\section{Competing interests}

The authors declare that they have no competing interests.

Received: 16 June 2016 Accepted: 31 October 2016

Published online: 21 November 2016

\section{References}

1. M Song, $C X i n, Y Z$ Zhao, $X$ Cheng, Dynamic spectrum access: from cognitive radio to network radio. IEEE Wirel. Commun. 19(1), 23-29 (2012)

2. E Ahmed, A Gani, S Abolfazli, LU Yao, SU Kahn, Channel assignment algorithms in cognitive radio networks: taxomony, open issues, and challenges. IEEE Commun. Surv. Tutor. 18(1), 795-823 (2016)

3. A Ahmad, S Ahmad, MH Rehmani, NU Hassan, A survey on radio resource allocation in cognitive radio sensor networks. IEEE Commun. Surv. Tutor. 17(2), 888-917 (2015)

4. AK Mir, A Akram, E Ahmed, J Qadir, A Baig, in Unified channel assignment for unicast and broadcast traffic in cognitive radio networks. Proceeding of the 12th IEEE International Workshop on Wireless Local Networks (WLN), (Clearwater, FL, USA, 2012), pp. 799-806

5. M Talal, E Ahmed, J Qadir, A Baig, in Quantifying the multiple cognitive radio interfaces advantage. Proceedings of 6th International Conference on Signal Processing and Communication Systems, (Gold Coast, Australia, 2012), pp. 12-14

6. A Bhattacharya, R Ghosh, K Sinha, D Datta, BP Sinha, Multimedia channel allocation in cognitive radio networks using FDMFDMA and OFDM-FDMA. CoRR, vol. abs/1603, 03938 (2016). http://arxiv.org/abs/1603.03938

7. VK Tumuluru, P Wang, D Niyato, W Song, Performance analysis of cognitive radio spectrum access with prioritized traffic. IEEE Trans. Veh. Technol. 61(4), 1895-1906 (2012)

8. TMC Chu, H Phan, H-J Zepernick, Dynamic spectrum access for cognitive radio network with prioritized traffics. IEEE Commun. Lett. 18(7), 1218-1221 (2014)

9. J-M Chung, CH Kim, J-H Park, J Shin, D Kim, Optimal channel allocation for cognitive radios. IET Electron Lett. 46(11), 802-803 (2010)

10. E Ahmed, I Qadir, A Baig, High-throughput transmission-quality-aware broadcast routing in cognitive radio networks. Wirel. Netw. 21(4), 1193-1210 (2015)

11. A Bhattacharya, R Ghosh, K Sinha, D Datta, BP Sinha, Noncontiguous channel allocation for multimedia communication in cognitive radio networks. IEEE Trans. Cog. Commun. Netw. 1(4), 420-434 (2015)

12. J-H Park, J-M Chung, Enhanced channel access estimation based adaptive control of distributed cognitive radio networks. KSII Trans. Internet Inf. Syst. 10(3), 1333-1343 (2016)

13. F Saffre, RTateson, R Ghanea-Hercock, Reliable sensor networks using decentralised channel selection. Comput. Netw. 46(5), 651-663 (2004)

14. J-H Park, Y Nam, J-M Chung, Sustainability enhancement multihop clustering in cognitive radio sensor networks. Int J Distrib. Sens. Netw. 11(10 574340), 1-8 (2015)

15. A Ahmad, S Ahmad, MH Rehmani, NU Hassan, A survey on radio resource allocation in cognitive radio sensor networks. IEEE Commun. Surv. Tutor. 17(2), 888-917 (2015)

16. G GuR, S Bayhan, F Alagoz, Cognitive femtocell networks: an overlay architecture for localized dynamic spectrum access. IEEE Wirel. Commun. 17(4), 62-70 (2010)

17. AO Bicen, EB Pehlivanoglu, S Galmes, OB Akan, Dedicated radio utilization for spectrum handoff and efficiency in cognitive radio networks. IEEE Trans. Wirel. Commun. 14(9), 5251-5259 (2015)

18. VK Tumuluru, P Wang, D Niyato, A novel spectrum-scheduling scheme for multichannel cognitive radio network. IEEE Trans. Veh. Technol. 60(4), 1849-1858 (2011)

19. Q Liu, S Zhou, GB Giannakis, Queuing analysis with adaptive modulation and coding over wireless links: Cross-layer analysis and design. IEEE Trans. Wirel. Commun. 40(3), 1142-1153 (2005)

20. H Su, X Zhang, Cross-layer-based opportunistic MAC protocols for QoS provisionings over cognitive radio wireless networks. IEEE J. Sel. Areas Commun. 26(1), 118-129 (2008) 
21. H Jiang, P Wang, W Zhuang, A distributed channel access scheme with guaranteed priority and enhanced fairness. IEEE Trans. Wirel. Commun. 6(6), 2114-2125 (2007)

22. D Bertsekas, R Gallager, Data networks, 2nd Ed. (Prentice-Hall, USA, 1992). ISBN: 0132009161

23. A Alshamrani, X Shen, L-L Xie, QoS provisioning for heterogeneous services in cooperative cognitive radio networks. IEEE J. Sel. Area Commun. 29(4), 819-830 (2011)

24. D Kivanc, G Li, H Liu, Computationally efficient bandwidth allocation and power control for OFDMA. IEEE Trans. Wirel. Commun. 2(6), 1150-1158 (2003)

\section{Submit your manuscript to a SpringerOpen ${ }^{\circ}$ journal and benefit from:}

- Convenient online submission

- Rigorous peer review

- Immediate publication on acceptance

- Open access: articles freely available online

- High visibility within the field

- Retaining the copyright to your article

Submit your next manuscript at $\gg$ springeropen.com 